\title{
Learning of Sensory Sequences in Cerebellar Patients
}

\author{
Markus Frings, ${ }^{1}$ Raoul Boenisch, Marcus Gerwig, Hans-Christoph Diener, \\ and Dagmar Timmann
}

Department of Neurology, University of Duisburg-Essen, 45122 Essen, Germany

\begin{abstract}
A possible role of the cerebellum in detecting and recognizing event sequences has been proposed. The present study sought to determine whether patients with cerebellar lesions are impaired in the acquisition and discrimination of sequences of sensory stimuli of different modalities. A group of 26 cerebellar patients and 26 controls matched for age, sex, handedness, musicality, and level of education were tested. Auditory and visual sensory sequences were presented out of different sensory pattern categories (tones with different acoustic frequencies and durations, visual stimuli with different spatial locations and colors, sequential vision of irregular shapes) and different ranges of inter-cue time intervals (fast and slow). Motor requirements were small, with vocal responses and no time restrictions. Perception of visual and acoustic stimuli was generally preserved in patients and controls. The number of errors was significantly higher in the faster tempo of sequence presentation in learning of sequences of tones of different frequencies and in learning of sequences of visual stimuli of different spatial locations and different colors. No difference in tempo between the groups was shown. The total number of errors between the two groups was identical in the sequence conditions. No major disturbances in acquisition or discrimination of various sensory sequences were observed in the group of cerebellar patients. Sequence learning may be impaired only in tasks with significant motor demands.
\end{abstract}

Thomas Willis (1664) was the first investigator to go beyond pure philosophic conjecture and anatomic observation about the function of the cerebellum. He tried to prove by clinical observations, comparative anatomical studies, and animal lesion studies that the cerebellum was the seat of involuntary movements. Marie-Jean-Pierre Flourens (1824) corrected this hypothesis by systematic animal lesion studies and supposed the cerebellum to be the organ for coordination of voluntary movements, but not a center for the production of movements. Our knowledge about the clinical manifestations of cerebellar dysfunction are based mainly on the investigations by Gordon Holmes of cerebellar patients with tumors (Holmes and Stewart 1904) and with gunshot wounds from World War I (Holmes 1917). In the most recent decades, an increasing number of studies reported evidence of involvement of the cerebellum not only in motor performance but also in motor learning, although the exact role of the cerebellum is still a matter of discussion (Thach 1996; Llinas et al. 1997). An additional contribution of the cerebellum to mental skills has been proposed (Leiner et al. 1986; Schmahmann 1991). This turn of research to cognitive functions of the cerebellum is a rediscovery of a concept from ancient history. The vermis of the cerebellum was considered to act as a valve in the ventricular system to regulate the flow of animal spirit. This theory became less important after the beginning of experimental research in the sixteenth century (Clarke and O'Malley 1996).

A strong anatomical argument in recent times for viewing the cerebellum as a motor structure was the concept that the cerebellar's output to the cerebrum is sent exclusively to a single motor cerebral cortical area (Asanuma et al. 1983). Several years ago, projections of the cerebellum to many different "nonmotor" cortical areas, including the frontal lobe, were discovered (Middleton and Strick 1997). Another reason for the motor theory is that most deficits associated with cerebellar dysfunction are motor (Dow and Moruzzi 1958). On the other hand, the

\footnotetext{
${ }^{1}$ Corresponding author.

E-MAIL markus.frings@uni-essen.de; FAX 49201 723-5901.

Article and publication are at http://www.learnmem.org/cgi/doi/10.1101/ Im.66904.
}

cerebellum receives sensory information of nearly every sensory modality for adjusting the motor output (Bloedel and Courville 1981). Several recent neuroimaging studies have demonstrated that the cerebellum is even strongly activated during sensory tasks when they are dissociated from motor behavior. A functional MRI (fMRI) study examined active and passive sensory tasks that human subjects performed with their fingers. Activation of the cerebellar dentate nucleus could be demonstrated during the acquisition and discrimination of sensory information, but no cerebellar activation could be found by control of movement per se (Gao et al. 1996). Moreover, a cerebellar contribution to visual and acoustic sensory information processing could be demonstrated. A PET study of mental rotation of abstract visual images in the absence of motor activity has revealed cerebellar activations (Parsons et al. 1995). Discrimination of tone intervals of different durations revealed activations of the cerebellar vermis and hemispheres bilaterally (Jueptner et al. 1995).

Braitenberg (1967) was the first to note that the cerebellar role in information processing of sensory stimuli may be judging the timing of events. Subjects with cerebellar lesions are impaired in the discrimination of the duration of the time interval between pairs of tones (Ivry and Keele 1989) and in estimating the velocity of dots moving on a screen (Ivry and Diener 1991). More precisely, the role of the cerebellum in perception of temporal characteristics of sensory patterns seems to be the correct sequencing of incoming stimuli and outgoing responses (Braitenberg et al. 1997; Ivry 1997; Mauk et al. 2000).

Therewith, deficits of cerebellar patients in sensory information processing on the one hand and in timing of events on the other hand have been suggested by numerous studies. These are two aspects of learning of sequences of sensory stimuli, which was examined by Shimansky et al. (1997). Human subjects were required to perceive an irregular two-dimensional shape by integrating temporal sequences of kinesthetic cues received through active or passive tracing of the shape while blindfolded. The capacity to discriminate a reference template from three others was severely impaired in cerebellar patients under both active and 
passive tracing conditions. A less severe deficit was found when the irregular shape was presented to the subject as a temporal sequence of visual cues generated by exposing only a small region of the shape at one time. In addition to an impairment of cerebellar patients in the perception based on kinesthetic cues, a reduced capacity to integrate temporal sequences of visual cues into a complete image was suspected. In the present study, we attempted to determine whether the use of sequence information is a cerebellar-dependent process in general. Therefore, different types of visual and auditory sensory modalities were addressed.

An involvement of the cerebellum in procedural learning of visuomotor sequences has been shown in human subjects by several studies (Molinari et al. 1997; Gomez-Beldarrain et al. 1998). However, it is difficult to dissociate motor functions from learning functions in a motor-learning paradigm. In a recent fMRI study of a serial reaction-time task, subjects performed a concurrent distractor task to suppress the performance changes associated with learning. No cerebellar activation associated with the learning phase was found, but the cerebellum seemed to contribute to the modification of motor performance after removal of the distractor (Seidler et al. 2002). In the present study, to exclude a motor dependency of a potential cerebellar involvement in sequence learning, motor demands were reduced as far as possible. Motor requirements were restricted to vocal responses. No time restrictions were given, and no reaction times were measured.

We compared the capacity of both patients with cerebellar dysfunction and that of normal human subjects to acquire a certain sequence of sensory stimuli and to discriminate this sequence from other sequences of the same type. Auditory and visual sensory modalities with different sensory pattern categories were employed in five paradigms (Figs. 1 and 2): tones with different acoustic frequencies (conditions of this paradigm are abbreviated by F) and durations (D), visual stimuli with different spatial locations (L) and colors (C), and sequential vision of irregular shapes $(\mathrm{V})$. Each paradigm consisted of two conditions with slower (the second letter of the abbreviations of these conditions is $\mathrm{S}$ ) or faster (F) tempo of sequence presentation, and a control condition (C) where stimuli were presented individually to control possible deficits in visual and acoustic perception. In the last paradigm, subjects had to learn an irregular shape (sets of shapes in Fig. 3) by sequential vision of line segments (as an exception abbreviated by VL) or single dots (VD). In the control condition, the shapes were visualized in its entirety (VC). Each condition consisted of four blocks in which five test sequences had to be discriminated from the reference sequence, which was
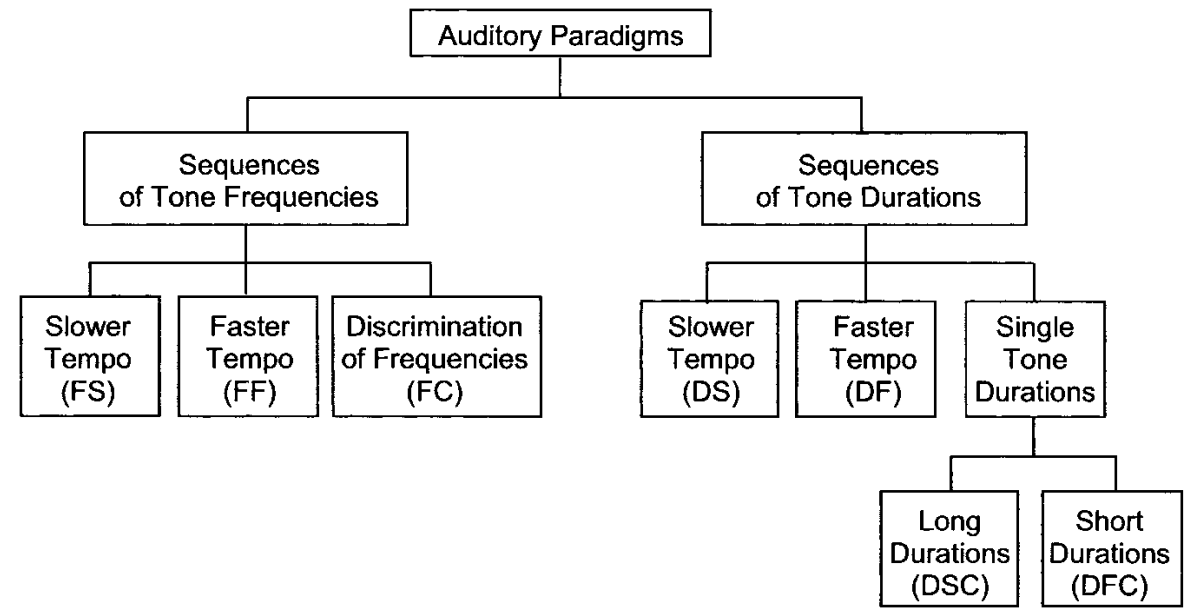

Figure 1 Test and control conditions of paradigms 1 and 2 (learning of sequences of acoustic tones). the same over all blocks. If the use of sequence information is cerebellar-dependent, the number of errors made by cerebellar patients should be higher than the number of errors made by the control group. An improvement of performance over the four blocks of each condition should be impaired in cerebellar patients. Moreover, the learning disability of cerebellar patients should be more pronounced in the faster tempo of sequence presentation.

\section{RESULTS}

\section{Learning of Sequences of Acoustic Tones}

The results of all conditions of the first paradigm "learning of sequences of tones of different frequencies" (F) are shown in Figure $4 \mathrm{~A}$. The total numbers of errors of both types (failure to recognize the reference pattern and identification of a nonreference pattern as the reference) made by the cerebellar patients $(n=26$, Groups 1 and 2 of Table 1$)$ and control subjects $(n=26)$ are presented for each of the four blocks. Error bars indicate the standard deviation. A univariate repeated-measures analysis was calculated using the number of errors as dependent variable, block number (blocks 1-4) and tempo (slow vs. fast conditions) as within-subject factors, and group (patients vs. controls) as between-subject factor. Probability of zero hypothesis was selected $P<0.05$ as the basis for determining significance. In order to control for multiple comparisons, Bonferroni adjustments were applied with the significance level set to $<0.01$ $(0.05 / 5$ paradigms). In the slower (FS) and faster (FF) tempo condition, the total number of errors made by the cerebellar patients was not significantly higher than the number of errors made by the control subjects (group effect: $P=0.42$ ). The total number of errors made in condition FF was significantly higher than that made in condition FS (tempo effect: $P<0.001$ ), and a trend for improvement of performance over the four blocks was observed (block effect: $P=0.012$ ). No significant tempo $\times$ group, block $\times$ group, or tempo $\times$ block $\times$ group interaction effects were found (all $P$-values $>0.3$ ). In the control condition FC, a significant block effect $(P<0.001)$ but no significant group or block $\times$ group interaction effects (all $P$-values $>0.08$ ) were found.

The results of the second paradigm "learning of sequences of tones of different durations" (D) are shown in Figure 4B. Twelve patients (Group 1 in Table 1 ) and 12 control subjects participated in this paradigm. In the slower (DS) and faster (DF) tempo conditions, there were no significant differences between groups, no significant tempo or block effects, and no tempo $\times$ group, block $\times$ group, or tempo $\times$ block $\times$ group interaction effects (all $P$-values $>0.08$ ). The mean error in duration perception (control conditions with discrimination of longer and shorter tone durations; DSC and DFC) was higher in cerebellar patients compared to controls, but did not reach significance $(P=0.02)$. Post hoc analyses revealed a significant group effect in condition DFC $(P=0.003)$, but not in condition DSC $(P=0.14)$. No significant block or tempo, tempo $\times$ group, or block $\times$ group interaction effects were observed (all $P$-values $>0.1$ ), but a significant tempo $\times$ block $\times$ group interaction effect $(P=0.002)$ was revealed, indicating that the cerebellar patients were starting from a higher level of errors to

\section{Learning \& Memory}

www.learnmem.org 


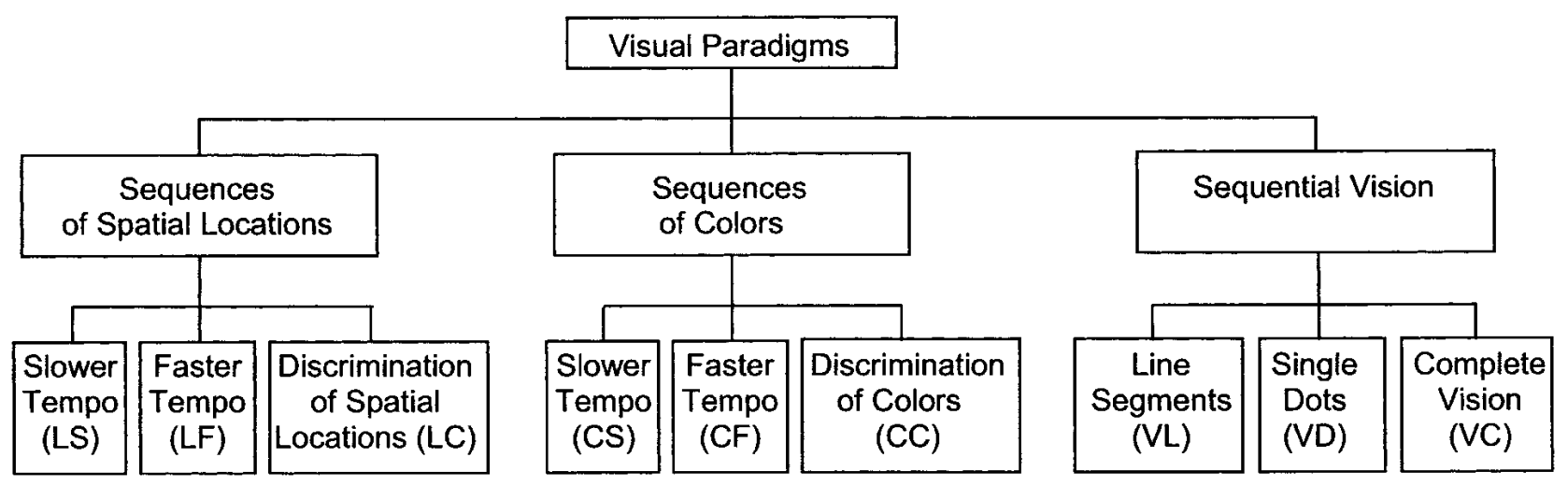

Figure 2 Test and control conditions of paradigms 3, 4, and 5 (learning of sequences of simple visual images).

reach the same level as the control subjects over the four blocks in the slower tempo condition, but not in the faster tempo condition.

Additionally, the two kinds of possible errors were analyzed separately. The number of errors that were made when the wrong template was recognized as an original showed a significant tempo effect in the first paradigm "learning of sequences of tones of different frequencies" $(P<0.001)$, but not in the second paradigm "learning of sequences of tones of different durations", similar to the analysis described above. No significant group, block, tempo $\times$ group, tempo $\times$ block, or tempo $\times$ block $\times$ group interaction effects were found (first paradigm: all $P$-values $>0.19$, second paradigm: all $P$-values $>0.28$ ). The number of errors that were made when the original templates were not recognized showed a significant block effect in the first paradigm $(P<0.005)$. No significant group, tempo $\times$ group, tempo $\times$ block, or tempo $\times$ block $\times$ group interaction effects and in the second paradigm also no tempo effect were found (first paradigm: all $P$-values $>0.09$, second paradigm: all $P$-values $>0.08$ ).

\section{Line Segments (VL)}

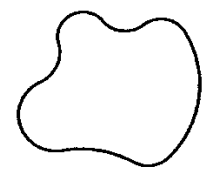

1

Single Dots (VD)

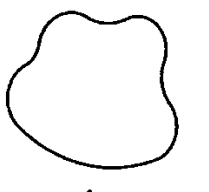

1

Complete Vision (VC)

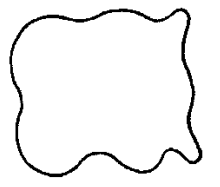

1

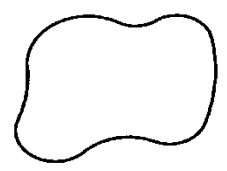

2

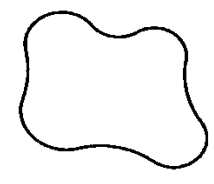

2

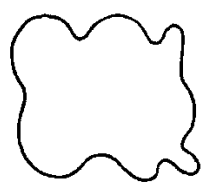

2
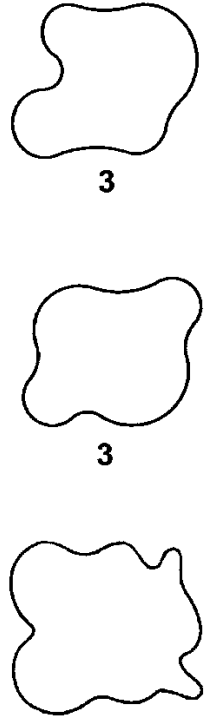

3

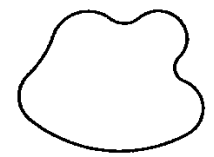

4

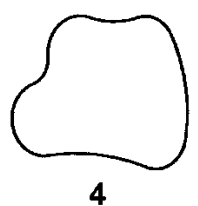

4

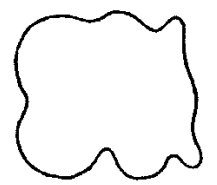

4
Figure 3 Three sets of shapes used in paradigm 5 (learning of templates by sequential vision). The first line shows the shapes of the condition sequential vision of lines (VL). The second line shows the shapes of the condition sequential vision of dots (VD). The third line shows the shapes of the control condition complete vision (VC). Shape 1 of each set of shapes was the reference shape; the others were test shapes.
To examine whether the degree of cerebellar symptoms had ationship with the performance of the cerebellar patients in digm was calculated using the total number of errors as dependent variable, block number and tempo as within-subject factors, and the severity of symptoms (groups of patients with mild, found. No significant group, block, tempo $\times$ group, were found (all $P$-values $>0.12$ ). In the second paradigm, there $\times$ block $\times$ group interaction effects were found (all for the slower or faster tempo condition of this paradigm (slower tempo: all $P$-values $>0.34$, faster tempo: all $P$-values $>0.05$ ). Additionally, a linear regression analysis of the number of errors across the four blocks for each condition and for each patient was performed. The linear correlation between the slope of the number of errors across the four blocks and the total score of ataxia scale of each patient was calculated. No significant correlations could be shown (all $P$-values $>0.08$ ).

\section{Learning of Sequences of Simple} Visual Images

Twelve patients (Group 1 in Table 1) and 12 matched control subjects participated in the third paradigm "learning of sequences of visual stimuli of different spatial locations" (L, see Fig. 5A). The total number of errors made in the faster tempo condition (LF) was significantly higher than that made in the slower tempo condition (LS, $P<0.001$ ), but no significant group effect $(P=0.56)$, a tendency of improvement of performance over the four blocks $(P=0.029)$, and no significant interaction effects (all $P$-values $>0.08$ ) were observed. Post hoc analyses revealed a significant block effect in condition LS $(P<0.001)$, but not in condition LF $(P>0.87)$. In the control condition LC, no significant group or block effect and no block $\times$ group interaction could be found $(P>0.2)$.

In the fourth paradigm "learning of se- 

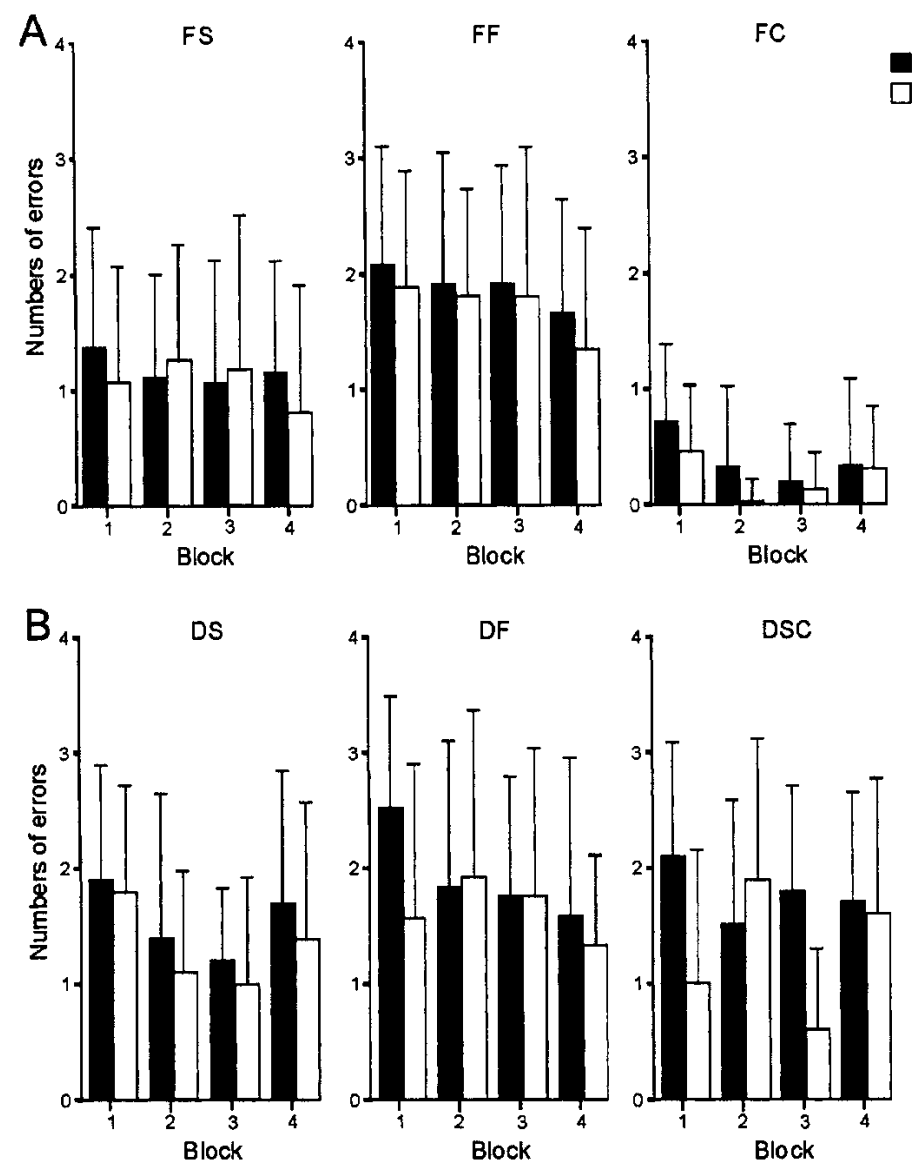

Figure 4 Learning of sequences of acoustic tones. $(A)$ Learning of sequences of tones of different frequencies with a slower tempo of successive tones (FS), faster tempo (FF), and the control condition (discrimination of tone frequencies, FC). Error rate per block (1-4) for all cerebellar patients $(n=26)$ and control subjects $(n=26)$. Error bars indicate the standard deviation. (B) Learning of sequences of tones of different durations $(n=12)$ with a slower tempo of successive tones (DS), faster tempo (DF), and the two control conditions [discrimination of long (DSC) and short (DFC) tone durations].

quences of different colors" (C), the total number of errors made by the cerebellar patients $(n=12$, Group 1 in Table 1$)$ in the slower (CS) and faster (CF) tempo conditions was not significantly higher than the total number of errors made by the 12 matched control subjects $(P=0.11$, see Fig. $5 \mathrm{~B})$, but the total number of errors made by patients and controls in condition CF was significantly higher than that made in condition CS $(P<0.001)$. A block effect close to being significant was found $(P=0.014)$. The tempo $\times$ block $\times$ group interaction effect $(P=0.013)$ showed a trend too, indicating that the improvement of performance over the four blocks was better in the group of cerebellar patients in the slower tempo condition. Control subjects performed worse in the first block of the faster tempo condition compared to patients. No significant tempo $\times$ group or block $\times$ group interaction effects were observed (all $P$-values $>0.19)$. In the control condition CC no significant group, block, or block $\times$ group interaction effects could be found $(P>0.28)$.

Fourteen patients (Group 2 in Table 1) and 14 control subjects participated in the fifth paradigm "learning of templates by sequential vision" (V, see Fig. 5C). No significant group effect and no significant difference in the total number of errors in the conditions "sequential vision of lines" (VL) and "sequential vision of dots" (VD) could be found $(P>0.21)$. A tendency of improvement of performance over the four blocks in VL rather than in VD was shown (block-effect: $P=0.022$, tempo $\times$ block inter-

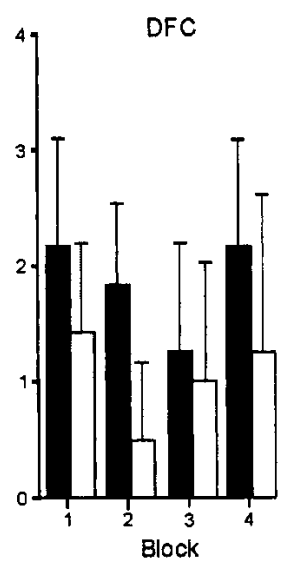

action: $P=0.025)$. Post hoc analyses revealed a significant block effect in condition VL $(P<0.001)$, but not in condition VD $(P=0.53)$. No significant tempo $\times$ group or tempo $\times$ block $\times$ group interactions were observed (all $P$-values $>0.13$ ). In the control condition $\mathrm{VC}$, no significant group, block, or block $\times$ group interaction effects could be found (all $P$-values $>0.03$ ).

By analyzing separately the number of errors that were made when the wrong template was recognized as an original, a significant tempo effect in the third and the fourth paradigms (for all paradigms $P<0.001)$ was observed. No significant group, block, tempo $\times$ group, tempo $\times$ block, or tempo $\times$ block $\times$ group interaction effects and in the fifth paradigm also no tempo effects were found (third paradigm: all $P$-values $>0.2$, fourth paradigm: all $P$-values $>0.18$, fifth paradigm: all $P$-values $>0.13)$. The number of errors that were made when the original templates were not recognized showed a significant block effect in the third, fourth, and fifth paradigm (for all paradigms $P<0.005)$. In the fourth paradigm, a significant tempo effect could be shown $(P=0.001)$. In no paradigm was a significant group, tempo $\times$ group, tempo $\times$ block, or tempo $\times$ block $\times$ group interaction effects found (third paradigm: all $P$-values $>0.27$, fourth paradigm: all $P$ values $>0.06$, fifth paradigm: all $P$-values $>0.07$ ).

The examination of the relationship between the degree of cerebellar symptoms and the performance of the cerebellar patients in the tasks showed a significant tempo effect $(P=0.003)$ in the fourth paradigm. In the third paradigm in the faster tempo condition, the number of errors was higher as in the slower tempo, but did not reach significance $(P=0.03)$. No significant group, block, tempo $\times$ group, tempo $\times$ block, or tempo $\times$ block $\times$ group interaction effects were found (third paradigm: all $P$-values $>0.37$, fourth paradigm: all $P$-values $>0.06)$. In the fifth paradigm, the tempo $\times$ block interaction effect showed a trend $(P=0.014)$ but did not reach significance. No significant tempo, group, block, tempo $\times$ group, or tempo $\times$ block $\times$ group interaction effects were found (all $P$-values $>0.08$ ). In a linear regression analysis of the number of errors across the four blocks and the total score of ataxia scale of each patient, a significant correlation was not observed for any condition (all $P$-values $>0.12$ ).

Plots of data (Figs. 4,5) suggested that cerebellar patients were worse than controls in the first block of trials. Therefore, the possible group effect of the first block of each condition was analyzed by an unpaired t-test. In the conditions DF and CS, a trend could be shown $(P=0.04$ and $P=0.03)$. No significant differences were found between cerebellar patients and controls in all other conditions (all other $P$-values $>0.28$ ).

\section{DISCUSSION}

In this study the expected impairment of cerebellar patients in learning of sequences of acoustic and visual stimuli could not be 
Table 1. Clinical Characteristics of Cerebellar Patients

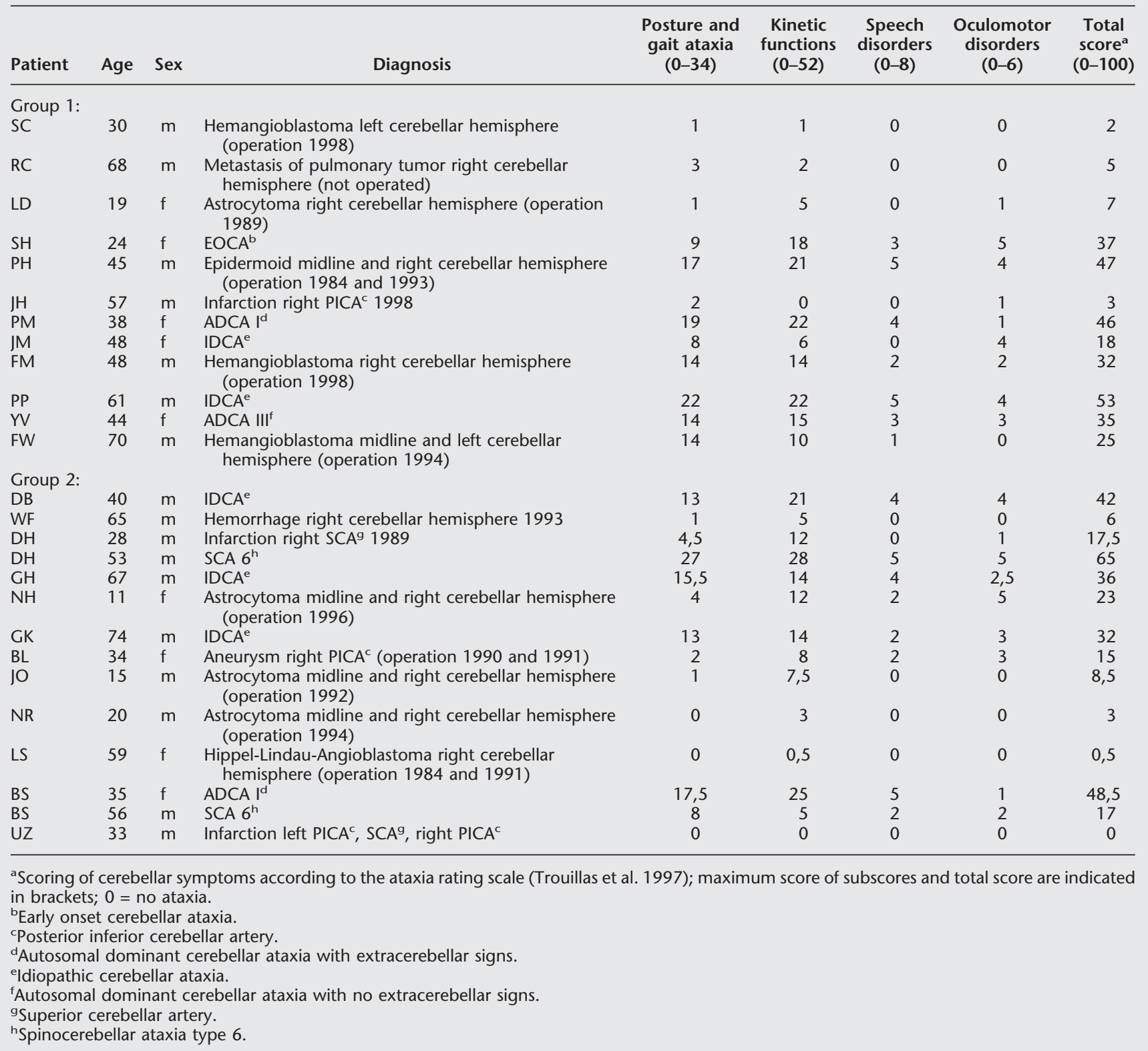

confirmed. In all conditions, where learning of acoustic and visual sequences was tested, no significant differences in numbers of errors between the cerebellar and control group could be found. Improvement of performance over the four blocks (i.e., learning) as well as tempo effects did not differ significantly between the cerebellar and control groups. In the control conditions, cerebellar-dependent deficits in acoustic or visual perception could be excluded, except for an impaired perception of tone durations.

\section{Learning of Sequences of Acoustic Tones}

In the present study, both paradigms "learning of sequences of tones of different frequencies" and "learning of sequences of tones of different durations" consisted of a slower and faster tempo condition. As expected, in the first paradigm the number of errors was significantly higher in the faster tempo condition, but no significant differences between cerebellar subjects and controls could be shown. However, in the control condition of the second paradigm, testing the perception of tone durations, in the faster tempo a significant difference in the total number of errors between cerebellar subjects and controls was found. The finding of disordered perception of tone durations in this control condition agrees with previous studies: Ivry and Keele (1989) showed an impairment in cerebellar patients in production and discrimination of time intervals of about $400 \mathrm{msec}$. The production task was to maintain a simple rhythm in finger-tapping. In the motor-independent discrimination task, subjects had to judge whether the duration of a time interval between a pair of tones was either longer or shorter than the reference duration of 400 msec. Likewise, a PET study of the discrimination of tone intervals of durations shorter or longer than $300 \mathrm{msec}$ showed activations of the cerebellar vermis and both cerebellar hemispheres (Jueptner et al. 1995). Disorders in the present study, however, were less severe compared to previous PET and lesion 

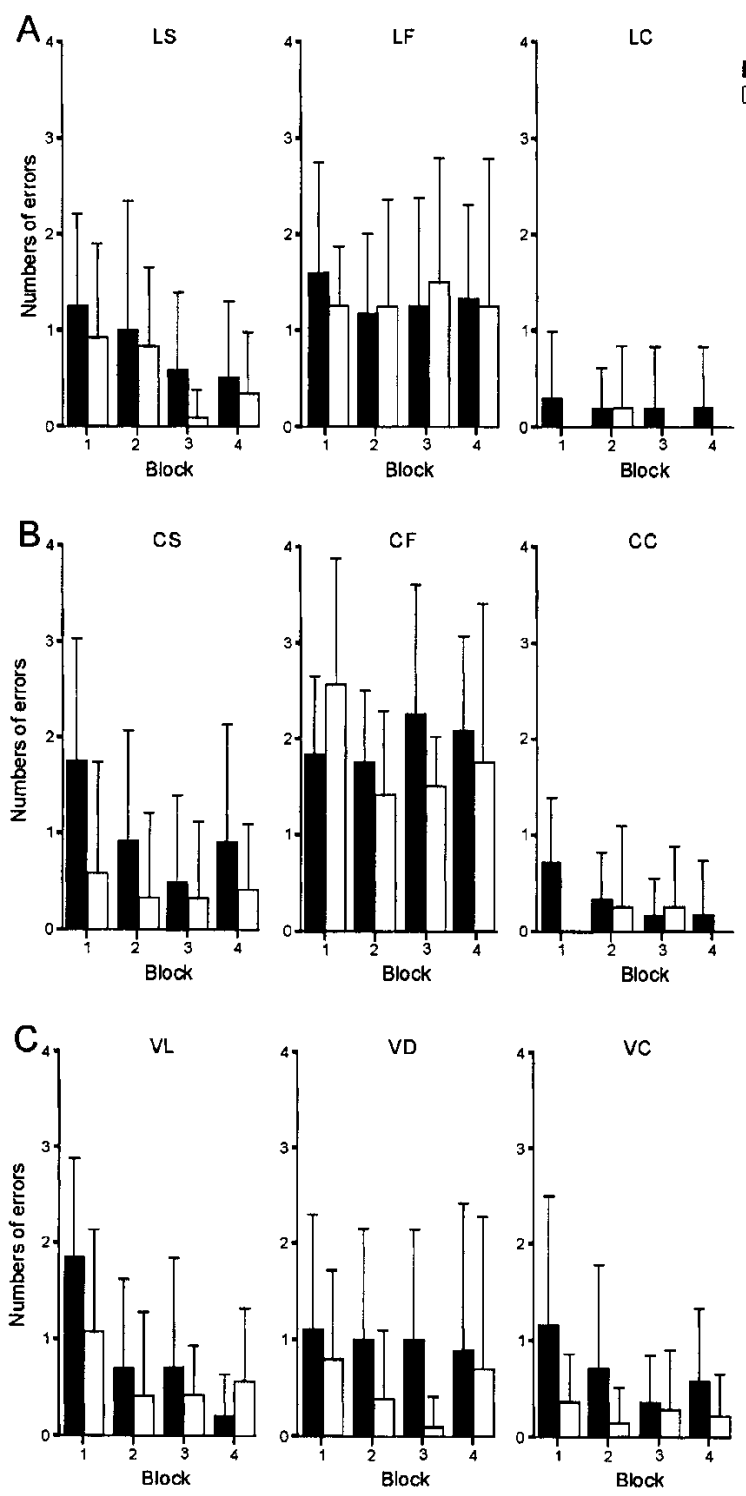

Figure 5 Learning of sequences of simple visual images. ( $A$ ) Learning of sequences of visual stimuli of different spatial locations $(n=12)$ with a slower tempo (LS), faster tempo (LF), and the control condition (discrimination of spatial locations, LC). (B) Learning of sequences of different colors $(n=12)$ with a slower tempo $(C S)$, faster tempo (CF), and the control condition (discrimination of different colors, CC). (C) Learning of templates by sequential vision $(n=14)$ with sequential vision of line segments (VL), sequential vision of single dots (VD), and the control condition (complete vision, $\mathrm{VC}$ ).

studies and present only at shorter time intervals (100 msec). In another study, fMRI was performed during silent repetition of the syllable "ta" at three different rates: $2.5,4.0$, and $5.5 \mathrm{~Hz}$ (Wildgruber et al. 2001). Similar to the present findings, cerebellar responses were restricted to fast performance.

In contrast to the control condition of the first paradigm of the present study Parsons (2001) reported an impairment of cerebellar patients in pitch discrimination. Subjects had to decide whether a comparison tone was higher or lower than a $500-\mathrm{Hz}$ standard. The pitch discrimination threshold was significantly higher for cerebellar patients than for control subjects. The disparity between the two studies could be explained by the fact that in the present study, frequencies of $500 \mathrm{~Hz}$ and above were applied, but no frequencies below $500 \mathrm{~Hz}$. In addition, in the present study the ability to differentiate between six frequencies was tested, and a discrimination threshold as such was not defined.

One possible explanation for the lack of cerebellar involvement in learning the sequences of different frequencies and durations may be that they could acquire an internal representation of the tone sequences as a melody and therefore discriminate them. As well as in the visual paradigms, other reasons may account for preserved sequence acquisition and discrimination in cerebellar patients: First, subjects could have been able to distinguish the sequences by memorizing; for example, only the first and last tone of the sequence instead of memorizing the whole sequence. Second, effects of learning (i.e., block effects) were generally weak. Parts of the paradigm may have been either too difficult or too easy for both patients and controls. Third, the group of cerebellar patients in the present study was quite heterogeneous, consisting of patients with focal lesions and degenerative cerebellar disorders. However, this heterogeneity should be compensated for by the considerable number of patients.

Another explanation may be that the present tasks had only small motor requirements. This will be discussed in more detail below.

\section{Learning of Sequences of Simple Visual Images}

In the slower tempo condition of the paradigm "learning of sequences of visual stimuli of different spatial locations" and in the condition "sequential vision of line segments" a significant improvement over the four blocks could be shown, but no differences between cerebellar subjects and controls in the total number of errors or in the effect of learning were observed. In the paradigms "learning of sequences of different colors" and "learning of sequences of visual stimuli of different spatial locations", the numbers of errors were significantly higher in the faster tempo condition as expected, but as in all other paradigms no significant differences between groups were observed. These results are at variance with previous studies. An involvement of the human cerebellum in procedural learning of visuomotor sequences by performing a serial reaction-time task was shown by Gomez-Beldarrain et al. (1998) and Molinari et al. (1997). Differences in findings between these studies and the present study may be explained by differences in motor demands of the tasks, that is, deficits of cerebellar patients in sequence learning may increase with increasing motor requirements. In the present study, the motor requirements of the tasks were restricted as far as possible. Instead of the pressing of a button and measurement of reaction times, learning was determined by vocal responses. Possible effects of dysarthria were minimized, because no time restrictions were given to the subjects. Although effects of oculomotor deficits cannot be excluded, motor requirements were less demanding compared to previous studies of learning of visuomotor sequences. Here, subjects had to press different response buttons for different visual items presented on a monitor (Molinari et al. 1997; Gomez-Beldarrain et al. 1998). Likewise, in previous studies of our group of stimulus-response and stimulusstimulus-response associations, subjects were required to release a rest button and push one of two response buttons (Drepper et al. 1999; Maschke et al. 2002; Timmann et al. 2002).

Other authors have suggested that the sensory input of the cerebellum may be most relevant for the control of movement (Seitz et al. 1991; Jueptner et al. 1997). This corresponds with recent findings of an fMRI imaging study of a serial reaction-time task that revealed a cerebellar activation associated with the modification of motor performance but not with the learning of the visuomotor skill itself (Seidler et al. 2002). Likewise, another 
fMRI study showed a cerebellar involvement in reassigning motor responses to different visual stimuli but not in switching attention between stimuli without motor demands (BischoffGrethe et al. 2002).

The study by Shimansky et al. (1997) is most closely related to the present study, and thus it will be discussed in more detail. The main finding in that study was that the cerebellar patients were impaired in perceiving an irregular two-dimensional shape by integrating temporal sequences of kinesthetic cues received through active or passive tracing of the shape while blindfolded. An impairment of cerebellar patients in their capability of perception based on kinesthetic cues was suspected. This is one possible reason for the differences from the present study, where no kinesthetic cues were required. A more recent study, however, provided evidence that cerebellar patients show no impairment in kinesthetic perception (Maschke et al. 2003). In the Shimansky et al. study, subjects could have developed an idea of the template by inner movement. Some previous studies suggest that cerebellar patients are impaired in motor imagery (Kagerer et al. 1998). If subjects have applied this strategy, it would be explainable that cerebellar patients were handicapped in the Shimansky et al. study and not worse than controls in the present study, where motor requirements were small and inner movement was not required.

In addition, in the Shimansky et al. study (1997), a reduced capacity to integrate temporal sequences of visual cues into a complete image was suspected even when no execution of movements was associated. In the Shimansky study, however, complex forms had to be integrated from sequential information, whereas in the present study only the order of sequences of stimuli had to be acquired. The condition "sequential vision of line segments" (VL) of the present study is the only one that is nearly similar to the condition "sequential vision" of the Shimansky et al. study. In contrast to the Shimansky study, in the condition VL of the present study the rotation time was shorter, the exposure of the shape at one time was larger, and the exposure of the segments of the shape was discontinuous. Therefore, it could have been easier for controls and cerebellar patients to acquire and discriminate the shapes so that no group effect could be found. Above all, in the condition "sequential vision" of the Shimansky study, the difference between patients and controls was very small and an effect could be shown only in post-hoc analysis. Lastly, differences in patient population may account for a part of the differences in results compared to the present study.

Finally, it should be noted that other studies suggest a role of the cerebellum in sensory information processing. Paulin (1993) showed that the development of specific cerebellar regions in some lower vertebrate species and mammals is strongly correlated with special needs in sensory information processing, rather than with the complexity of their motor behavior. Several recent neuroimaging studies have demonstrated that the cerebellum is activated during sensory tasks even when they are dissociated from motor behavior (Jueptner et al. 1995; Parsons et al. 1995; Gao et al. 1996). Furthermore, cerebellar lesions may cause sensory timing deficits (Ivry and Keele 1989; Ivry and Diener 1991). Parsons (2001) showed an impairment of cerebellar patients in pitch discrimination. Thier et al. (1999) supported the existence of visual disturbances in cerebellar patients, primarily impaired motion perception. In a sequential speed discrimination task, however, that required storage of visual information for a later comparison with other patterns, cerebellar patients showed no impairment. These findings agree with the present findings of unimpaired sensory sequence learning in cerebellar patients. The present study was not designed to show small differences in perception of sensory stimuli between groups. Rather, differences between single sensory stimuli of one modality were comparatively large, to allow a significant amount of sequence learning in controls.

No clear impairment of cerebellar patients in learning of sequences of acoustic and visual stimuli was observed in the present study. A cerebellar role in sensory sequence learning may become evident only if the sequence information has to be connected with a significant motor response.

\section{MATERIALS AND METHODS}

\section{Subjects}

The study was performed in 26 cerebellar patients and 26 healthy subjects. All subjects were without any hearing or visual loss, and not receiving any medication. Patients and controls were matched for age, sex, handedness, musicality, and level of education. The mean age of the patients was $43.9 \pm 18.3$ (range 1174 ) years with an estimated mean duration of disease of 8.8 years; the mean age of the control subjects was $44.4 \pm 19.2$ (range 1177) years. Only patients with pathology restricted to the cerebellum were selected for the study. The neurological examination included the scoring of cerebellar ataxia according to Trouillas et al. 1997 (clinical data in Table 1). There were nine patients with no or mild signs of cerebellar ataxia (total ataxia score $<10$ out of 100 ), four with moderate signs of cerebellar ataxia (total ataxia score 10-20), and 13 patients with marked signs of cerebellar ataxia (total ataxia score $>20$ ). All subjects gave informed written consent to testing. The study was approved by the local ethical committee.

\section{Basic Experimental Procedure}

The ability to acquire sequences of sensory stimuli of acoustic and visual modalities was tested. Acquisition was quantified by the ability of the subjects to differentiate reference sequences from other sequences of the same stimulus modality. Sequences of acoustic tones were tested in two paradigms (Fig. 1): In the first paradigm, tones of a sequence differed in their frequency (conditions of this paradigm are abbreviated by F); in the second paradigm, they differed in their duration (D). The capacity to perform the acquisition and discrimination of sequences of simple visual images was tested in three paradigms (Fig. 2): In the first paradigm, the stimuli differed in their spatial location $(\mathrm{L})$; in the second paradigm, they differed in their color (C). Thirdly, templates had to be learned by sequential vision (V). Each paradigm consisted of two conditions with slower (the second letter of the abbreviations of these conditions is S) or faster (F) tempo of sequence presentation. In a control condition (C), stimuli were presented individually to control possible deficits in visual and acoustic perception.

Each condition consisted of four blocks. At the beginning of each block, the subject was instructed to attend to and memorize the reference sequence, which was presented three times. The reference sequence was the same for all four blocks of a certain condition. Subjects were aware of that fact. Next, in each block, five test sequences were presented in a pseudorandom order, each of them three times in a row. Two of the test sequences were the same as the reference sequence, and the other three were different from it and from each other. After the presentation of each test sequence, the subject was required to indicate whether it was identical to the reference sequence or not. To minimize motor components, the subjects were instructed to use the short words "yes" and "no."

Tasks were presented on a 15 -inch color monitor placed 60 $\mathrm{cm}$ in front of the comfortably seated subject. The background color on the monitor was black. The subjects' answers were recorded in a protocol form by the experimenter. The reaction time was not measured. The average duration of each condition was 5 $\min$. 


\section{Learning of Sequences of Acoustic Tones}

\section{Paradigm 1: Learning of Sequences of Tones of Different Frequencies}

Subjects had to learn the sequence of six different tones. Frequencies were 500, 741, 982, 1223, 1464, and $1705 \mathrm{~Hz}$ with nonharmonic differences between them. Tones were presented without a pause between them or an overlap in time. Subjects had to differentiate the reference sequence, which was the same for all four blocks, from other sequences of the same tones (test sequences). As explained above, there were five test sequences, two of which were identical to the reference sequence, in each of four blocks. Each of the sequences was presented three times in a row. There were three different conditions. The duration of the time interval between the onsets of successive tones, which is equal to the duration of one tone, was $400 \mathrm{msec}$ (slower tempo condition, FS) and $100 \mathrm{msec}$ (faster tempo condition, FF), respectively. The frequencies were the same, but the order of the tones differed in the reference and the test sequences in both conditions. To control for subjects being able to perceive the frequencies of the tones, they were presented separately and with a duration of 100 msec (control condition, FC). In accord with the other conditions, the reference tone was the same for all four blocks. Two of the test tones were the same as the reference tone, and the other three were different from it and from each other. The acoustic tones were generated by the computer.

\section{Paradigm 2: Learning of Sequences of Tones of Different Durations}

In the second paradigm, different tone durations were used as the distinctive feature of the acoustic tones in each sequence. In the first condition (slower tempo condition, DS), $1000-\mathrm{Hz}$ tones of three different durations $(260,380$, and $480 \mathrm{msec})$ were employed. The subjects were required to perform the acquisition/ discrimination of the sequences of six such tones with a 70-msec inter-tone pause. Each duration was represented twice in each sequence. Neither the reference sequence nor the three test sequences had an internal regularity reducing their complexity. The design of the second condition (DF) was the same as the design of the first one, except that acoustic tones of much shorter durations $(80,140$, and $190 \mathrm{msec})$ were used to form sequences of a faster tempo. The inter-tone pause in this task was $40 \mathrm{msec}$. In the two control conditions, the subjects performed the acquisition/discrimination of single tones with different durations instead of sequences of tones. The reference duration was one of the three long (DSC) or short (DFC) durations. It was the same for all four blocks. The reference duration and one of the other two durations were represented twice in the block of the five test durations.

\section{Learning of Sequences of Simple Visual Images}

\section{Paradigm 3: Learning of Sequences of Visual Stimuli of Different Spatial Locations}

In this paradigm the subjects were required to perform the acquisition/discrimination of sequences of six different spatial locations of a yellow-filled square $(20 \mathrm{~mm} \times 20 \mathrm{~mm})$ displayed on the computer screen. The six spatial locations were arranged as the vertices of a regular hexagon with a $40-\mathrm{mm}$ radius and its center located at the center of the screen. Every sequence included all of these locations (once each). In each sequence, the images were displayed in a consecutive manner, with neither a pause between the exposure offset of one image and the exposure onset of the subsequent image nor a time overlap of their exposures. In the first condition, the inter-image time interval was $400 \mathrm{msec}$ (LS), in the second $120 \mathrm{msec}$ (LF). In the control condition (LC), instead of a sequence of spatial locations, the yellow square was presented in only one spatial location to the subject. Unlike all preceding test and control conditions, in which the reference stimulus was the same in all four trial blocks, in this control condition the reference location was different among the four blocks. Otherwise it would have been too easy for the subjects to discriminate reference and test stimuli.

\section{Paradigm 4: Learning of Sequences of Different Colors}

In the next paradigm, cerebellar patients and control subjects performed acquisition/discrimination of the temporal order of colors. Filled squares $(20 \mathrm{~mm} \times 20 \mathrm{~mm})$ of six different colors (blue, cyan, green, magenta, red, and yellow) were presented at the center of a computer screen one after another without a pause. All of these colors were included (once each) in the reference sequence and each test sequence. There were two conditions: fast tempo (CF) and slower tempo condition (CS) with color display durations of 170 and $500 \mathrm{msec}$, respectively. In the control condition (CC), the different colors had to be discriminated. Instead of a color sequence, only one color was presented as reference and test stimulus. As in the control condition of the third paradigm, the reference color was different across the four blocks.

\section{Paradigm 5: Learning of Templates by Sequential Vision}

In the last paradigm, subjects had to learn the shape of a twodimensional irregularly shaped trace by sequential vision of line segments (VL) or single dots (VD). The shapes had an average height of $14 \mathrm{~cm}$. Figure 3 shows the sets of shapes of the VL and VD conditions and the control condition. For each condition, shape 1 was the reference shape, and the other three shapes were test shapes. The line segments and dots were generated consecutively by a computer, without a pause between them or an overlap in time and without a spatial overlap of the exposures of the shape. In the VL condition, $10 \%$ of the outline of the shape was exposed at any one time. One full turn was made in $5 \mathrm{sec}$ in the VL condition, and in $15 \mathrm{sec}$ in the VD condition. Subjects could examine the shapes of reference and test sequences during three full turns in a row. In the control condition, the shapes were visualized in its entirety (VC). The degree of template shape complexity was slightly higher than in the conditions VL and VD. In the control condition, reference and test shapes were demonstrated on a sheet of paper instead of a computer monitor. Subjects had $20 \mathrm{sec}$ to learn the reference shape, which was the same for all four blocks, before consecutive presentation of the five test sequences, two of which were the same as the reference sequence, as in all other conditions of the study.

The order of the conditions in the experimental procedure was LS, LF, LC, DS, DSC, CS, CF, CC, DF, DFC, FS, FF, FC (for explanation see Figs. 1, 2) for 12 patients (Group 1 in Table 1) and control subjects. For another 14 patients (Group 2 in Table 1) and control subjects, the order was VL, FS, FF, FC, VD, VC.

\section{Data Analysis}

Numbers of errors (failure to recognize the reference sequences and identification of a nonreference sequence as the reference) made in each block were determined. For each paradigm, a univariate repeated measures analysis was calculated using the number of errors as dependent variable, block number (block 1-4) and tempo (slow vs. fast conditions) as within-subject factors, and group (patients vs. controls) as between-subject factor. Total numbers of errors and the two kinds of possible errors were analyzed separately for each paradigm. In the fifth paradigm, the conditions VL (vision of line segments) and VD (vision of single dots) were compared according to the comparison between the slower or faster tempo condition in the other paradigms. Probability of zero hypothesis was selected $P<0.05$ as the basis for determining significance. In order to control for multiple comparisons, Bonferroni adjustments were applied with the significance level set to $<0.01$ ( $0.05 / 5$ paradigms).

To compare the performance of both groups in the control condition of each paradigm, a univariate repeated-measures analysis was calculated with the number of errors as dependent variable, block number as within-subject factor, and group as between-subject factor. To compare the performance of both groups in the two control conditions of the second paradigm, a univariate repeated-measures analysis was calculated with the number of errors as dependent variable, block number and tempo as within-subject factor, and group as between-subject factor. 
To examine whether there was a relationship between the severity of cerebellar symptoms and the performance of the patients in the tasks, a univariate repeated-measures analysis using the severity of symptoms (three groups of mild, moderate, and severe ataxia) as between-subject factor was performed. Further, a linear correlation between the slope of the number of errors across the four blocks of each condition and the total score of ataxia scale of each patient was calculated.

\section{ACKNOWLEDGMENTS}

The study was supported by a grant from the Deutsche Forschungsgemeinschaft (DFG Ti 239/2-3, 7-1). We thank Y. Shimansky, Department of Bioengineering, Arizona State University, for the development of the experimental paradigms and for sharing the software with our laboratory. We thank B. Brol and H.G. Elles for technical assistance.

The publication costs of this article were defrayed in part by payment of page charges. This article must therefore be hereby marked "advertisement" in accordance with 18 USC section 1734 solely to indicate this fact.

\section{REFERENCES}

Asanuma, C., Thach, W.T., and Jones, E.G. 1983. Distribution of cerebellar terminations and their relation to other afferent terminations in the ventral lateral thalamic region of the monkey. Brain Res. Rev. 5: 237-265.

Bischoff-Grethe, A., Ivry, R.B., and Grafton, S.T. 2002. Cerebellar involvement in response reassignment rather than attention. $J$. Neurosci. 22: 546-553.

Bloedel, J.R. and Courville, J. 1981. A review of cerebellar afferent systems. In Handbook of physiology, motor control (ed. V.B. Brooks), Vol. II, pp. 735-830. Williams and Wilkins, Baltimore, MD.

Braitenberg, V. 1967. Is the cerebellar cortex a biological clock in the millisecond range. Progr. Brain Res. 25: 334-346.

Braitenberg, V., Heck, D., and Sultan, F. 1997. The detection and generation of sequences as a key to cerebellar function: Experiments and theory. Behav. Brain Sci. 20: 229-245.

Clarke, E. and O'Malley, C.D. 1996. The human brain and spinal cord, 2nd rev. ed., pp. 628-707. Norman Publishing, San Francisco.

Dow, R.S. and Moruzzi, G. 1958. The physiology and pathology of the cerebellum. University of Minnesota Press, Minneapolis, MN

Drepper, J., Timmann, D., Kolb, F.P., and Diener, H.C. 1999. Non-motor associative learning in patients with isolated degenerative cerebellar disease. Brain 122: 87-97.

Fluorens, M.-J.-P. 1824. Recherches expérimentales sur les propriétés et les fonctions du système nerveux dans les animaux vertébrés. J.B. Ballière, Paris.

Gao, J.H., Parsons, L.M., Bower, J.M., Xiong, J., Li, J., and Fox, P.T. 1996. Cerebellum implicated in sensory acquisition and discrimination rather than motor control. Science 272: 545-547.

Gomez-Beldarrain, M., Garcia-Monco, J.C., Rubio, B., and Pascual-Leone, A. 1998. Effect of focal cerebellar lesions on procedural learning in the serial reaction time task. Exp. Brain Res. 120: 25-30.

Holmes, G. 1917. The symptoms of acute cerebellar injuries due to gunshot injuries. Brain 40: 461-535.

Holmes, G. and Stewart, T.G. 1904. Symptomatology of cerebellar tumours: A study of forty cases. Brain 27: 522-591.

Ivry, R. 1997. Cerebellar timing systems. Int. Rev. Neurobiol. 41: 555-573.

Ivry, R.B. and Diener, H.C. 1991. Impaired velocity perception in patients with lesions of the cerebellum. J. Cogn. Neurosci. 3: 355-366.

Ivry, R.B. and Keele, S.W. 1989. Timing functions of the cerebellum. J. Cogn. Neurosci. 1: 136-152.

Jueptner, M., Rijntjes, M., Weiller, C., Faiss, J.H., Timmann, D., Müller S.P., and Diener, H.C. 1995. Localization of a cerebellar timing process using PET. Neurology 145: 1540-1545.

Jueptner, M., Ottinger, S., Fellows, S.J., Adamschewski, J., Flerich, L., Müller, S.P., Diener, H.C., Thilmann, A.F., and Weiller, C. 1997. The relevance of sensory input for the cerebellar control of movements. Neuroimage 5: 41 .

Kagerer, F.A., Bracha, V., Wunderlich, D.A., Stelmach, G.E., and Bloedel, J.R. 1998. Ataxia reflected in the simulated movements of patients with cerebellar lesions. Exp. Brain Res. 121: 125-134

Leiner, H.C., Leiner, A.L., and Dow, R.S. 1986. Does the cerebellum contribute to mental skills? Behav. Neurosci. 100: 443-454.

Llinas, R., Lang, E.J, and Welsh, J.P. 1997. The cerebellum, LTD, and memory: Alternative views. Learn. Mem. 3: 444-445.

Maschke, M., Drepper, J., Bürgerhoff, K., Calabrese, S., Kolb, F.P., Daum, I., Diener, H.C., and Timmann, D. 2002. Differences in trace and delay visuomotor associative learning in cerebellar patients. Exp. Brain Res. 147: 538-548.

Maschke, M., Gomez, C.M., Tuite, P.J., and Konczak, J. 2003. Dysfunction of the basal ganglia, but not the cerebellum, impairs kinaesthesia. Brain 126: 2312-2322.

Mauk, M.D., Medina, J.F., Nores, W.L., and Ohyama, T. 2000. Cerebellar function: Coordination, learning or timing? Curr. Biol. 10: R522-R525.

Middleton, F.A. and Strick, P.L. 1997. Dentate output channels: Motor and cognitive components. Progr. Brain Res. 114: 553-566.

Molinari, M., Leggio, M.G., Solida, A., Corra, R., Misciagna, S., Silveri, M.C., and Petrosini, L. 1997. Cerebellum and procedural learning: Evidence from focal cerebellar lesions. Brain 120: 1753-1762.

Parsons, L.M. 2001. Exploring the functional neuroanatomy of music performance, perception, and comprehension. Ann. N.Y. Acad. Sci. 930: $211-231$.

Parsons, L.M., Fox, P.T., Downs, J.H., Glass, T., Hirsch, T.B., Martin, C.C., Jerabek, P.A., and Lancaster, J.L. 1995. Use of implicit motor imagery for visual shape discrimination as revealed by PET. Nature 375: $54-58$

Paulin M.G. 1993. The role of the cerebellum in motor control and perception. Brain Behav. Evol. 41: 39-50.

Schmahmann, J.D. 1991. An emerging concept. The cerebellar contribution to higher function. Arch. Neurol. 48: 1178-1187.

Seidler, R.D., Purushotham, A., Kim, S.-G., Ugurbil, K., Willingham, D. and Ashe, J. 2002. Cerebellum activation associated with performance change but not motor learning. Science 296: 2043-2046.

Seitz, R.J., Roland, P.E., Bohm, C., Greitz, T., and Stone-Elander, S. 1991 Somatosensory discrimination of shape: Tactile exploration and cerebral activation. Eur. J. Neurosci. 3: 481-492.

Shimansky, Y., Saling, M., Wunderlich, D.A., Bracha, V., Stelmach, G.E. and Bloedel, J.R. 1997. Impaired capacity of cerebellar patients to perceive and learn two-dimensional shapes based on kinesthetic cues. Learn. Mem. 4: 36-48.

Thach, W.T. 1996. On the specific role of the cerebellum in motor learning and cognition: Clues from PET activation and lesion studies in man. Behav. Brain Sci. 19: 411-431.

Thier, P., Haarmeier, T., Treue, S., and Barash, S. 1999. Absence of a common functional denominator of visual disturbances in cerebellar disease. Brain 122: 2133-2146.

Timmann, D., Drepper, J., Maschke, M., Kolb, F.P., Böring, D., Thilmann, A.F., and Diener, H.C. 2002. Motor deficits cannot explain impaired cognitive associative learning in cerebellar patients. Neuropsychologia 40: 788-800.

Trouillas, P., Takayanagi, T., Hallett, M., Currier, R.D., Subramony, S.H. Wessel, K., Bryer, A., Diener, H.C., Massaquoi, S., Gomez, C.M., et al 1997. International Cooperative Rating Scale for pharmacological assessment of the cerebellar syndrome. J. Neurol. Sci. 145: 205-211.

Wildgruber, D., Ackermann, H., and Grodd, W. 2001. Differential contributions of motor cortex, basal ganglia, and cerebellum to speech motor control: Effects of syllable repetition rate evaluated by fMRI. Neuroimage 13: 101-109.

Willis T. 1664. Cerebri anatome: Cui accessit nervorum descriptio et usus. Martin and Allestry, London. (Translation by R. Tercentenary, 1964. Thomas Willis, The anatomy of the brain and the nerves. McGill University Press, Montreal).

Received July 9, 2003; accepted in revised form March 16, 2004. 


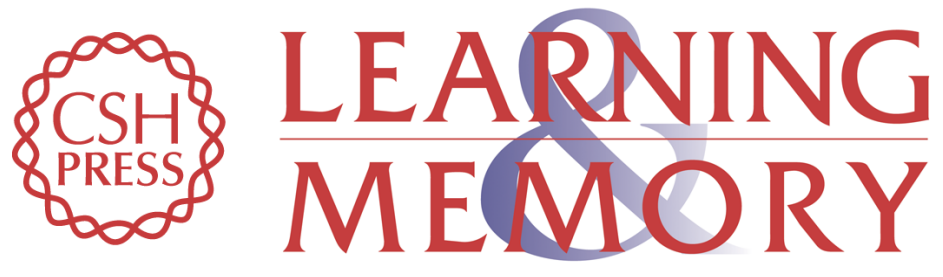

\section{Learning of Sensory Sequences in Cerebellar Patients}

Markus Frings, Raoul Boenisch, Marcus Gerwig, et al.

Learn. Mem. 2004, 11:

Access the most recent version at doi:10.1101/lm.66904

References This article cites 33 articles, 4 of which can be accessed free at: http://learnmem.cshlp.org/content/11/3/347.full.html\#ref-list-1

License

Email Alerting Receive free email alerts when new articles cite this article - sign up in the box at the Service top right corner of the article or click here. 